\title{
Asymptotics of the Stirling numbers of the first kind revisited: A saddle point approach
}

\section{Guy Louchard}

Université Libre de Bruxelles, Dép. d'Informatique, CP 212, B-1050 Bruxelles, Belgium. Iouchard@ulb.ac.be

received April 11, 2009, revised Dec. 21, 2009, accepted Feb. 18, 2010.

Using the saddle point method, we obtain from the generating function of the Stirling numbers of the first kind $\left[\begin{array}{c}n \\ j\end{array}\right]$ and Cauchy's integral formula, asymptotic results in central and non-central regions. In the central region, we revisit the celebrated Goncharov theorem with more precision. In the region $j=n-n^{\alpha}, \quad \alpha>1 / 2$, we analyze the dependence of $\left[\begin{array}{l}n \\ j\end{array}\right]$ on $\alpha$.

Keywords: Stirling numbers, saddle point method.

To Philippe

\section{Introduction}

Let $\left[\begin{array}{l}n \\ j\end{array}\right]$ be the Stirling number of the first kind (unsigned version). Their generating function is given by

$$
\phi_{n}(z)=\prod_{0}^{n-1}(z+i)=\frac{\Gamma(z+n)}{\Gamma(z)}, \quad \phi_{n}(1)=n !
$$

In the sequel all asymptotics are meant for $n \rightarrow \infty$.

An asymptotic expansion for $j=\mathcal{O}(1)$ is given in Wilf [14], which has been extended to the range $j=\mathcal{O}(\ln n)$ by Hwang [6]. The generalized Stirling numbers have been considered by Tsylova [13] and Chelluri et al. [2]. The $q-$ Stirling numbers are studied in Kyriakoussis and Vamvakari [9].

In Sec 2, we revisit the asymptotic expansions in the central region and in Sec 3, we analyse the noncentral region $j=n-n^{\alpha}, \quad \alpha>1 / 2$. We use Cauchy's integral formula and the saddle point method. 


\section{Central region}

Consider the random variable $J_{n}$, with probability distribution

$$
\begin{aligned}
\mathbb{P}\left[J_{n}=j\right]=Z_{n}(j), \\
Z_{n}(j):=\frac{\left[\begin{array}{l}
n \\
j
\end{array}\right]}{n !} .
\end{aligned}
$$

The mean and variance are given by

$$
\begin{aligned}
M & :=\mathbb{E}\left(J_{n}\right)=\sum_{0}^{n-1} \frac{1}{1+i}=H_{n}=\psi(n+1)+\gamma \\
\sigma^{2} & :=\mathbb{V}\left(J_{n}\right)=\sum_{0}^{n-1} \frac{i}{(1+i)^{2}}=\psi(1, n+1)+\psi(n+1)-\frac{\pi^{2}}{6}+\gamma,
\end{aligned}
$$

where $\psi(x)$ is the digamma function, $\psi(k, x)$ is the $k$ th polygamma function, and

$$
\begin{aligned}
M & \sim \ln (n)+\gamma+\frac{1}{2 n}+\mathcal{O}\left(\frac{1}{n^{2}}\right), \\
\sigma^{2} & \sim \ln (n)-\frac{\pi^{2}}{6}+\gamma+\frac{3}{2 n}+\mathcal{O}\left(\frac{1}{n^{2}}\right) .
\end{aligned}
$$

It is convenient to set

$$
A_{n}:=\ln (n)-\frac{\pi^{2}}{6}+\gamma=\ln \left(n e^{\gamma-\pi^{2} / 6}\right),
$$

and to consider all our next asymptotics $(n \rightarrow \infty)$ as functions of $A_{n}$. Of course, all asymptotics can be reformulated in terms of $\ln (n)$.

We have

$$
\begin{aligned}
M & \sim A_{n}+\frac{\pi^{2}}{6}+\mathcal{O}\left(\frac{1}{n}\right), \\
\sigma^{2} & \sim A_{n}+\mathcal{O}\left(\frac{1}{n}\right) .
\end{aligned}
$$

A celebretated central limit theorem of Goncharov says that

$$
J_{n} \sim \mathcal{N}(M, \sigma),
$$

where $\mathcal{N}$ is the Gaussian distribution, with a rate of convergence $\mathcal{O}(1 / \sqrt{\ln (n)})$. This can also be deduced from the Quasi-Power theorem of Hwang [7],[8].

In this Section, we want to obtain a more precise local limit theorem for $J_{n}$ in terms of $x:=\frac{J_{n}-M}{\sigma}$ and $A_{n}$. Actually, we obtain the following theorem, where we use $B_{n}:=\sqrt{A_{n}}$ to simplify the expressions. 


\section{Theorem 2.1}

$$
\begin{aligned}
Z_{n}(j) & \sim \frac{1}{\sqrt{2 \pi} B_{n}} e^{-x^{2} / 2} . \\
\cdot & {\left[1+\frac{x^{3} / 6-x / 2}{B_{n}}+\frac{3 x^{2} / 8-x^{4} / 6-1 / 12+x^{6} / 72}{B_{n}^{2}}\right.} \\
& \left.+\frac{-\pi^{2} x^{3} / 18+37 x^{5} / 240-355 x^{3} / 144+x / 8-x^{7} / 48+x^{9} / 1296+\pi^{2} x / 6-\zeta(3) x+\zeta(3) x^{3} / 3}{B_{n}^{3}}+\ldots\right] .
\end{aligned}
$$

Proof: By Cauchy's theorem,

$$
\begin{aligned}
Z_{n}(j) & =\frac{1}{2 \pi \mathbf{i}} \int_{\Omega} \frac{\phi_{n}(z)}{z^{j+1} n !} d z \\
& =\frac{1}{2 \pi \mathbf{i}} \int_{\Omega} e^{S(z)} d z
\end{aligned}
$$

where $\Omega$ is inside the analyticity domain of the integrand and encircles the origin and

$$
\left.S(z)=S_{1}(z)+S_{2}(z), \quad S_{1}(z)=\sum_{i=0}^{n-1} \ln (z+i)\right)-\ln (n !), \quad S_{2}(z)=-(j+1) \ln (z) .
$$

We will use the Saddle point method (for a good introduction to this method, see Flajolet and Sedgewick [3], ch.VIII). Set

$$
S^{(i)}:=\frac{d^{i} S}{d z^{i}} .
$$

These derivatives can be expressed in terms of $\psi(k, z+n)$ and $\psi(k, z)$.

First we must find the solution of

$$
S^{(1)}(\tilde{z})=0
$$

with smallest module.

Set $\tilde{z}:=z^{*}-\varepsilon$, where $z^{*}=\lim _{n \rightarrow \infty} \tilde{z}$. Here, it is easy to check that $z^{*}=1$. Set $j=M+x \sigma, x$ fixed and $B_{n}:=\sqrt{A_{n}}$.

This leads, to first order (keeping only the $\varepsilon$ term in (1)), to

$$
\varepsilon:=\frac{-x}{B_{n}}+\frac{x^{2}-1}{B_{n}^{2}}+\ldots+\frac{1}{n}\left(\frac{3 x}{4 B_{n}^{3}}+\ldots\right)+\mathcal{O}\left(\frac{1}{n^{2} B_{n}^{4}}\right) .
$$

This shows that, asymptotically, $\varepsilon$ is given by a series of powers of $n^{-1}$, where each coefficient is given by a series of powers of $B_{n}^{-1}$. To obtain more precision, we set again $j=M+x \sigma$, expand in powers of $n^{-1}$, and equate each coefficient to 0 . . This leads to (here and in the following, we provide only a few terms but Maple knows more)

$$
\varepsilon=\frac{-x}{B_{n}}-\frac{1}{B_{n}^{2}}+\frac{0}{B_{n}^{3}}+\ldots+\frac{1}{n}\left(\frac{3 x}{4 B_{n}^{3}}+\frac{x^{2}+3 / 2}{B_{n}^{4}}+\ldots\right)+\mathcal{O}\left(\frac{1}{n^{2} B_{n}^{4}}\right) .
$$


We have, with $\tilde{z}:=z^{*}-\varepsilon=1-\varepsilon$,

$$
Z_{n}(j)=\frac{1}{2 \pi \mathbf{i}} \int_{\Omega} \exp \left[S(\tilde{z})+S^{(2)}(\tilde{z})(z-\tilde{z})^{2} / 2 !+\sum_{l=3}^{\infty} S^{(l)}(\tilde{z})(z-\tilde{z})^{l} / l !\right] d z .
$$

Note that the linear term vanishes. Set $z=\tilde{z}+\mathbf{i} \tau$. This gives

$$
Z_{n}(j)=\frac{1}{2 \pi} \exp [S(\tilde{z})] \int_{-\infty}^{\infty} \exp \left[S^{(2)}(\tilde{z})(\mathbf{i} \tau)^{2} / 2 !+\sum_{l=3}^{\infty} S^{(l)}(\tilde{z})(\mathbf{i} \tau)^{l} / l !\right] d \tau
$$

The justification of the integration procedure is given in the Appendix. Let us first analyze $S(\tilde{z})$. We obtain

$$
\begin{aligned}
S(\tilde{z}) & =-x^{2} / 2+\frac{x^{3} / 6-x}{B_{n}}+\frac{-x^{4} / 12+x^{2} / 2-1 / 2}{B_{n}^{2}} \\
& +\frac{-x^{3} / 3+x^{5} / 20+x / 2-\pi^{2} x^{3} / 18+\zeta(3) x^{3} / 3}{B_{n}^{3}}+\ldots+\mathcal{O}\left(\frac{1}{n B_{n}^{3}}\right) .
\end{aligned}
$$

Also,

$$
\begin{aligned}
& S^{(2)}(\tilde{z})=B_{n}^{2}-B_{n} x-1+x^{2}+\ldots, \\
& S^{(3)}(\tilde{z})=-2 B_{n}^{2}+4 B_{n} x-\pi^{2} / 3+2 \zeta(3)-6 x^{2}+4+\ldots, \\
& S^{(4)}(\tilde{z})=6 B_{n}^{2}-18 B_{n} x+36 x^{2}-18+\pi^{2}-\pi^{4} / 15+\ldots, \\
& S^{(l)}(\tilde{z})=\mathcal{O}\left(B_{n}^{2}\right), l \geq 5 .
\end{aligned}
$$

We need these many terms in the following. Note that, with $z=\tilde{z} e^{\mathbf{i} \theta}$, this leads to

$$
S^{(2)}(\tilde{z}) \frac{(z-\tilde{z})^{2}}{2} \sim-\frac{1}{2} \ln (n) \theta^{2} .
$$

We can now compute (2), for instance by using the classical trick of setting

$$
S^{(2)}(\tilde{z})(i \tau)^{2} / 2 !+\sum_{l=3}^{\infty} S^{(l)}(\tilde{z})(i \tau)^{l} / l !=-u^{2} / 2 .
$$

Computing $\tau$ as a truncated series in $u$, this gives, by inversion,

$$
\tau=\left[u\left(1+x /\left(2 B_{n}\right)+\ldots\right)+u^{2}\left(\mathbf{i} /\left(3 B_{n}\right)+\ldots\right)+u^{3}\left(-1 /\left(36 B_{n}^{2}\right)+\ldots\right)\right] / B_{n}+\ldots
$$

Setting $d \tau=\frac{d \tau}{d u} d u$, expanding w.r.t. $B_{n}$ and integrating on $[u=-\infty . . \infty]$, this gives

$$
\frac{1}{\sqrt{2 \pi} B_{n}}\left[1+\frac{x}{2 B_{n}}+\frac{5 / 12-x^{2} / 8}{B_{n}^{2}}+\frac{x\left(8 \pi^{2}-10-93 x^{2}-48 \zeta(3)\right)}{48 B_{n}^{3}}+\ldots\right] .
$$




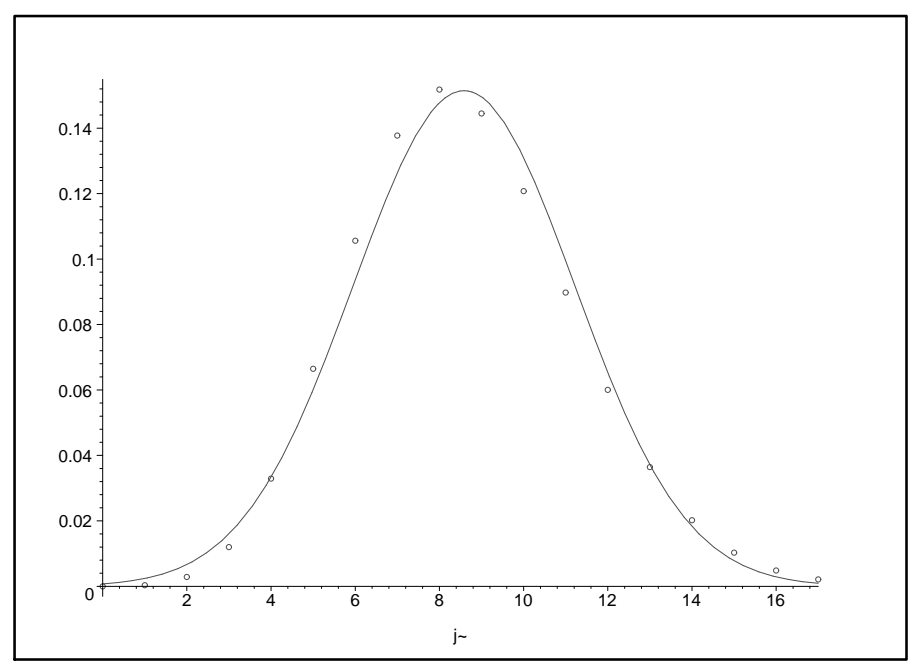

Fig. 1: Comparison between $Z_{n}(j)$ and $\frac{1}{\sqrt{2 \pi} \sigma} \exp \left[-\left(\frac{j-M}{\sigma}\right)^{2} / 2\right], n=3000$

Finally (2) leads to

$$
\begin{aligned}
Z_{n}(j) & \sim \frac{1}{\sqrt{2 \pi} B_{n}} e^{-x^{2} / 2} . \\
& \cdot \exp \left[\frac{x^{3} / 6-x}{B_{n}}+\frac{-x^{4} / 12+x^{2} / 2-1 / 2}{B_{n}^{2}}+\frac{-x^{3} / 3+x^{5} / 20+x / 2-\pi^{2} x^{3} / 18+\zeta(3) x^{3} / 3}{B_{n}^{3}}+\ldots\right] . \\
& \cdot\left[1+\frac{x}{2 B_{n}}+\frac{5 / 12-x^{2} / 8}{B_{n}^{2}}+\frac{x\left(8 \pi^{2}-10-93 x^{2}-48 \zeta(3)\right)}{48 B_{n}^{3}}+\ldots\right],
\end{aligned}
$$

or

$$
\begin{aligned}
Z_{n}(j) & \sim R_{1}, \\
R_{1} & =\frac{1}{\sqrt{2 \pi} B_{n}} e^{-x^{2} / 2} . \\
\cdot & {\left[1+\frac{x^{3} / 6-x / 2}{B_{n}}+\frac{3 x^{2} / 8-x^{4} / 6-1 / 12+x^{6} / 72}{B_{n}^{2}}\right.} \\
+ & \left.\frac{-\pi^{2} x^{3} / 18+37 x^{5} / 240-355 x^{3} / 144+x / 8-x^{7} / 48+x^{9} / 1296+\pi^{2} x / 6-\zeta(3) x+\zeta(3) x^{3} / 3}{B_{n}^{3}}+\ldots\right] .
\end{aligned}
$$

For $n=3000$, a comparison between $Z_{n}(j)$ and $\frac{1}{\sqrt{2 \pi} \sigma} \exp \left[-\left(\frac{j-M}{\sigma}\right)^{2} / 2\right]$ is given in Figure 1 . 


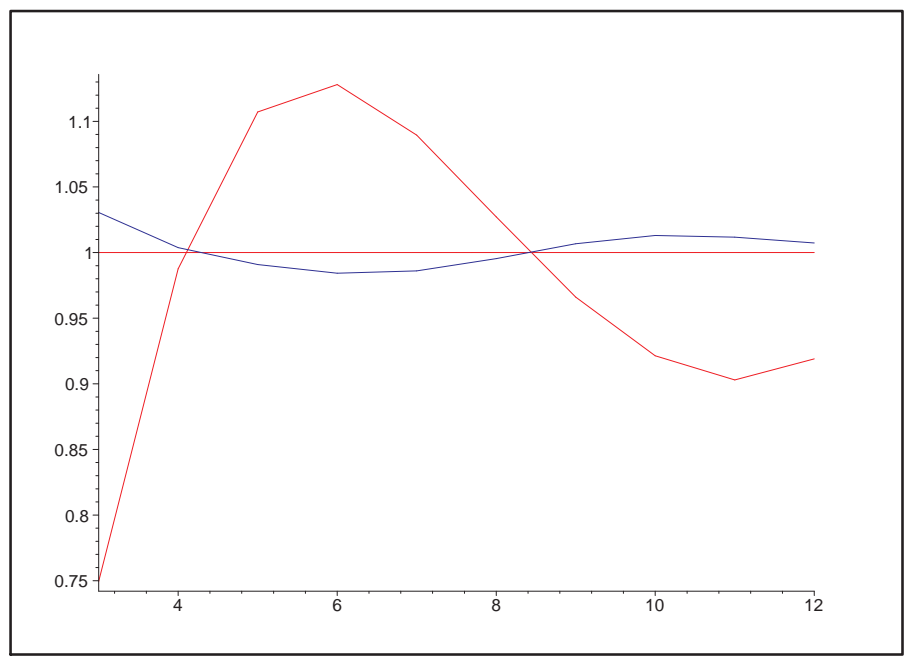

Fig. 2: $Z_{n}(j) /\left[\frac{1}{\sqrt{2 \pi} \sigma} \exp \left[-\left(\frac{j-M}{\sigma}\right)^{2} / 2\right]\right]$, color=red, $Z_{n}(j) / R_{1}$, color=blue, $n=3000$

Of course, only few values of $j$ are significant and also the quality of the Gaussian is low, all asymptotic expressions depend actually on powers of $A_{n}^{-1}$, but $A_{n}$ is not large.

A comparison of $Z_{n}(j) /\left[\frac{1}{\sqrt{2 \pi} \sigma} \exp \left[-\left(\frac{j-M}{\sigma}\right)^{2} / 2\right]\right]$ with $Z_{n}(j) / R_{1}$, with 2 terms in $R_{1}$, is given in Figure 2

The precision of $R_{1}$ is of order $10^{-2}$. Using 3 terms in $R_{1}$ leads to a less good result: $A_{n}$ is not large enough to take advantage of the $A_{n}^{-3 / 2}$ term: $A_{n}=6.94$ here, we deal with asymptotic series, not necessarily convergent ones. More terms can be computed in $R_{1}$ (which is almost automatic with Maple).

\section{Large deviation, $j=n-n^{\alpha}, \quad 1>\alpha>1 / 2$}

The case $j=\mathcal{O}(n)$ is analyzed in Timashev [12]. But he obtains a series of powers of $n^{-1}$, determined by a power series of a certain function that depends on the solution of a given non-linear differential equation of the first order. The coefficients obey some linear recurrence relations in the complex plane. The case $j=n-c, c$ constant, is considered in Grünberg [5]. As previous work for the case $j=n-n^{\alpha}$, let us mention Bender [1], Temme [11], Moser and Wyman [10] (see also the comments by Odlyzko in [4], p.1182). They all use, explicitly or not, the Saddle point method. For $\alpha<1 / 2$, Moser and Wyman (6.9) give an explicit asymptotic expression. For $\alpha>1 / 2$, they first compute in (4.52) the numerical solution $z n$ of $S^{\prime}(z n)=0$ and give in (4.51) an asymptotic expression. This is rather precise: for $n=50$, this gives a precision of order $10^{-4}$. [1] and [11] also compute numerically $z n$.

However, all these results do not shed light on the dependence of $\left[z^{j}\right] \phi(z)$ on $n^{\alpha}$. This is what we want 
to explicit in this Section. It appears that the range $\alpha>1 / 2$ is more delicate than the other range.

Recall that

$$
\phi_{n}(z)=\prod_{0}^{n-1}(z+i)=\frac{\Gamma(z+n)}{\Gamma(z)}
$$

We have

$$
G_{n}(z):=\frac{\Gamma(z+n)}{\Gamma(z) z^{j+1}}=\exp [S(z)]
$$

with

$$
S(z)=S_{1}(z)+S_{2}(z), S_{1}(z)=\sum_{0}^{n-1} \ln (z+i), S_{2}(z)=-(j+1) \ln (z) .
$$

We first compute $\tilde{z}$ such that

$$
S^{\prime}(\tilde{z})=0
$$

We have

$$
S^{\prime}(z)=\psi(z+n)-\psi(z)-\frac{j+1}{z} .
$$

Similarly (we need these expressions later on)

$$
\begin{aligned}
& S^{(2)}(z)=\psi(1, z+n)-\psi(1, z)+\frac{j+1}{z^{2}}, \\
& S^{(k)}(z)=\psi(k-1, z+n)-\psi(k-1, z)+(-1)^{k}(k-1) ! \frac{j+1}{z^{k}} .
\end{aligned}
$$

Some experiments with some values for $\alpha$ ( $\alpha=5 / 8$ is a good choice) show that $\tilde{z}$ must be a combination of $x=n^{\alpha}$ and $y=n^{1-\alpha}$ and $x \gg y \gg 1$. Note that both $x$ and $y$ are large. We will derive series of powers of $x^{-1}$, where each coefficient is a series of powers of $y^{-1}$. We obtain the following theorem

Theorem 3.1

$$
\begin{aligned}
{\left[z^{j}\right] \phi_{n}(z) } & \sim \frac{1}{\sqrt{2 \pi}} \frac{y^{2} \sqrt{x}}{2} \exp \left[x \left[1-\ln (2)+2 \ln (y)+\ln (x)-\frac{2}{3 y}-\frac{2}{9 y^{2}}-\frac{44}{405 y^{3}}-\frac{26}{405 y^{4}}+\frac{40}{27 y^{5}}\right.\right. \\
& \left.\left.+\frac{179968}{18225 y^{6}}+\frac{4727552}{127575 y^{7}}+\frac{3436796}{32805 y^{8}}+\frac{5492621728}{22143375 y^{9}}+\ldots\right]+\ln (2)-2 \ln (y)-\ln (x)\right] \\
\cdot & {\left[1-\frac{3}{3 y}-\frac{1}{18 y^{2}}-\frac{1}{30 y^{3}}+\frac{17207}{3240 y^{4}}+\ldots+\frac{1}{x}\left(-\frac{1}{12}+\frac{1}{36 y}-\frac{35}{216 y^{2}}+\frac{15029}{3240 y^{3}}+\ldots\right)\right.} \\
+ & \left.\frac{1}{x^{2}}\left(\frac{1}{288}-\frac{1}{864 y}+\frac{3527}{5184 y^{2}}+\ldots\right)+\mathcal{O}\left(\frac{1}{x^{3}}\right)\right]
\end{aligned}
$$

Proof: Let us summarize the different steps of the proof. First we compute $\tilde{z}$ and $S(\tilde{z})$ as $S(\tilde{z})=T_{1} T_{2}$, where $T_{1}$ is the dominant term and $T_{2}$ is a series of powers of $x^{-1}$, where each coefficient is a series of powers of $y^{-1}$. We expand $T_{3}:=e^{T_{2}}$. Next the integration procedure leads to $\frac{y^{2} \sqrt{x}}{2} T_{4}$, where $T_{4}$ is again a series of powers of $x^{-1}$, where each coefficient is a series of powers of $y^{-1}$. We set $T_{5}:=\frac{1}{\sqrt{2 \pi}} \frac{y^{2} \sqrt{x}}{2} e^{T_{1}}$. Finally, we obtain

$$
\left[z^{j}\right] \phi_{n}(z) \sim T_{5} T_{3} T_{4}
$$


The first terms in the asymptotics of $\tilde{z}$ are easy to compute: set $\tilde{z}=n \beta$. Equation (4) leads to

$$
\psi(n(1+\beta))-\psi(n \beta)=\frac{1}{\beta}-\frac{1}{y \beta}+\frac{1}{n \beta} .
$$

But $\psi(n) \sim \ln (n)$. So we have

$$
\ln \left(1+\frac{1}{\beta}\right) \sim \frac{1}{\beta}-\frac{1}{y \beta}+\frac{1}{n \beta},
$$

or

$$
\frac{1}{\beta}-\frac{1}{2 \beta^{2}} \sim \frac{1}{\beta}-\frac{1}{y \beta},
$$

or $\beta \sim \frac{y}{2}$.

More generally, we have

$\beta=\frac{y}{2}\left[1+\frac{a_{1}}{y}+\frac{a_{2}}{y^{2}}+\frac{a_{3}}{y^{3}}+\ldots+\frac{1}{x}\left(1+\frac{b_{1}}{y}+\frac{b_{2}}{y^{2}}+\ldots\right)+\frac{1}{x^{2}}\left(1+\frac{c_{1}}{y}+\frac{c_{2}}{y^{2}}+\ldots\right)+\mathcal{O}\left(\frac{1}{x^{3}}\right)\right]$.

Note that $\frac{1}{y^{3}}$ can be of the same order than $\frac{1}{x}$, see below.

By bootstrapping, we obtain (we give the first terms)

$$
\begin{aligned}
\tilde{z} & =\frac{n y}{2}\left[1-\frac{4}{3 y}+\frac{2}{9 y^{2}}+\frac{8}{135 y^{3}}+\frac{8}{405 y^{4}}+\frac{16}{1701 y^{5}}+\frac{232}{45525 y^{6}}+\frac{64}{18225 y^{7}}+\ldots\right. \\
& +\frac{1}{x}\left[1-\frac{1}{y}+\frac{4}{9 y^{2}}-\frac{16}{135 y^{3}}+\ldots\right] \\
& +\frac{1}{x^{2}}\left[1-\frac{1}{y}+\frac{0}{y^{2}}+\ldots\right] \\
& +\frac{1}{x^{3}}[1+\ldots] \\
& \left.+\mathcal{O}\left(\frac{1}{x^{4}}\right)\right] .
\end{aligned}
$$

Note that the choice of dominant terms in the bracket of 6 depends on $\alpha$. For instance, for $\alpha=3 / 4$, the dominant terms (in decreasing order) are

$$
1, \frac{1}{y}, \frac{1}{y^{2}},\left\{\frac{1}{x}, \frac{1}{y^{3}}\right\},\left\{\frac{1}{x y}, \frac{1}{y^{4}}\right\},\left\{\frac{1}{x y^{2}}, \frac{1}{y^{5}}\right\},\left\{\frac{1}{x^{2}}, \frac{1}{x y^{3}}, \frac{1}{y^{6}}\right\}, \ldots
$$

Now we must compute $S(\tilde{z})$ and its asymptotics. First we compute $\ln (\tilde{z}+i)$, take the asymptotics wrt $x$, sum on $i$, and again take the asymptotics wrt $x$ (recall that $n=x y$ ). this leads to

$$
\begin{aligned}
S_{1}(\tilde{z}) & =x\left[(-\ln (2)+2 \ln (y)+\ln (x)) y-\frac{1}{3}+\frac{4}{405 y^{2}}+\frac{2}{405 y^{3}}+\ldots\right]+y-\frac{2}{3}-\frac{2}{3 y}-\frac{49}{135 y^{2}}+\ldots \\
& +\frac{1}{x}\left(\frac{y}{2}+\frac{1}{6 y}+\ldots\right)+\frac{1}{x^{2}}\left(\frac{y}{3}+\ldots\right)+\mathcal{O}\left(\frac{y}{x^{3}}\right) .
\end{aligned}
$$




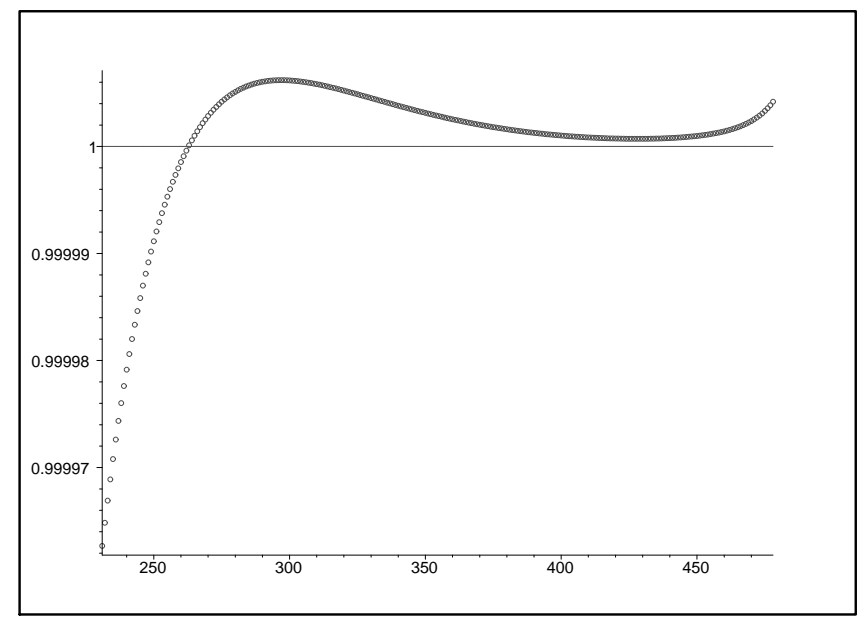

Fig. 3: $z n / \tilde{z}, n=500$, as function of $j$, full range

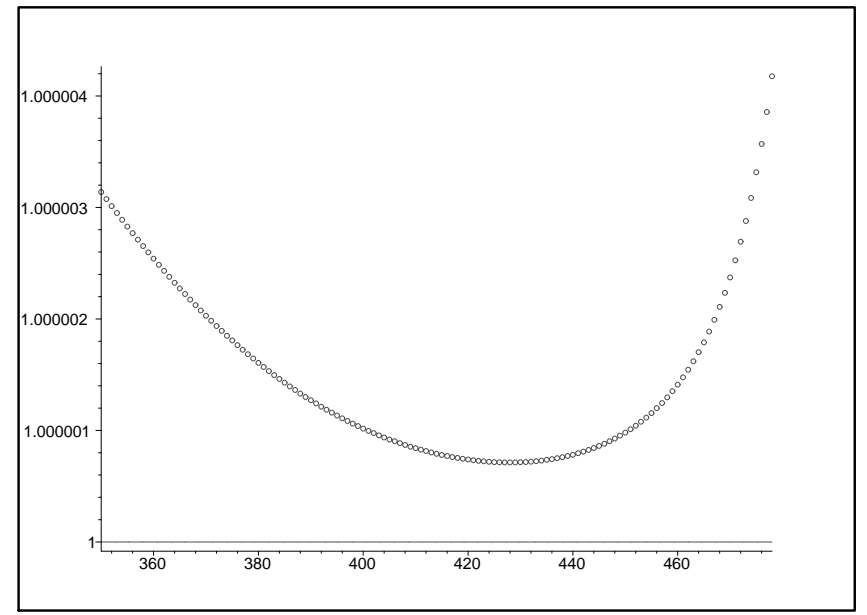

Fig. 4: $z n / \tilde{z}, n=500$, as function of $j$, restricted range 
Here we provide only a few terms but Maple knows more. Next

$$
\begin{aligned}
S_{2}(\tilde{z}) & =x\left[(\ln (2)-2 \ln (y)-\ln (x)) y+\frac{4}{3}-\ln (2)+2 \ln (y)+\ln (x)-\frac{2}{3 y}-\frac{94}{405 y^{2}}+\ldots\right] \\
& -y+\frac{2}{3}+\ln (2)-2 \ln (y)-\ln (x)+\frac{1}{y}+\frac{94}{135 y^{2}}+\ldots \\
& +\frac{1}{x}\left(\frac{y}{2}+\frac{1}{6 y}+\ldots\right) \\
& +\frac{1}{x^{2}}\left(\frac{y}{3}+\ldots\right) \\
& +\mathcal{O}\left(\frac{y}{x^{3}}\right) .
\end{aligned}
$$

So, finally

$$
\begin{aligned}
S(\tilde{z}) & =S_{1}(\tilde{z})+S_{2}(\tilde{z}) \sim x\left[1-\ln (2)+2 \ln (y)+\ln (x)-\frac{2}{3 y}+\ldots\right] \\
& +\ln (2)-2 \ln (y)-\ln (x)+\frac{1}{3 y}+\frac{1}{3 y^{2}}+\ldots \\
& +\frac{1}{x}\left(-\frac{1}{2}+\frac{1}{3 y}-\frac{1}{2 y^{2}}+\ldots\right) \\
& +\frac{1}{x^{2}}\left(-\frac{1}{6}+\frac{19}{18 y^{2}} \ldots\right) \\
& +\mathcal{O}\left(\frac{1}{x^{3}}\right) .
\end{aligned}
$$

Now we split $S(\tilde{z})$ into two parts:

$$
\begin{aligned}
T_{1} & =x\left[1-\ln (2)+2 \ln (y)+\ln (x)-\frac{2}{3 y}+\ldots\right]+\ln (2)-2 \ln (y)-\ln (x) \\
T_{2} & =\frac{1}{3 y}+\frac{1}{3 y^{2}}+\ldots \\
& +\frac{1}{x}\left(-\frac{1}{2}+\frac{1}{3 y}-\frac{1}{2 y^{2}}+\ldots\right) \\
& +\frac{1}{x^{2}}\left(-\frac{1}{6}-\frac{17}{18 y^{2}} \ldots\right) \\
& +\mathcal{O}\left(\frac{1}{x^{3}}\right)
\end{aligned}
$$

Note that the dominant term of $T_{1}$ is given by

$$
T_{1} \sim(2-\alpha) n^{\alpha} \ln (n) .
$$


We obtain

$$
\exp (S(\tilde{z}))=e^{T_{1}} e^{T_{2}}=e^{T_{1}} T_{3}
$$

with

$$
\begin{aligned}
T_{3} & =e^{T_{2}}=1+\frac{1}{3 y}+\frac{7}{18 y^{2}}+\frac{89}{270 y^{3}}+\frac{18263}{3240 y^{4}}+\frac{98009}{3240 y^{5}}+\frac{9517337}{97200 y^{6}}+\frac{491504273}{2041200 y^{7}}+\ldots \\
& +\frac{1}{x}\left(-\frac{1}{2}+\frac{1}{6 y}-\frac{7}{12 y^{2}}+\frac{2311}{540 y^{3}}+\frac{112469}{6480 y^{4}}+\frac{5137}{144 y^{5}}+\ldots\right) \\
& +\frac{1}{x^{2}}\left(-\frac{1}{24}-\frac{13}{72 y}-\frac{557}{932 y^{2}}+\ldots\right) \\
& +\mathcal{O}\left(\frac{1}{x^{3}}\right) .
\end{aligned}
$$

Here we have given all terms compatible with the expansion (6). Also, with more precision,

$$
\begin{aligned}
T_{1} & =x\left[1-\ln (2)+2 \ln (y)+\ln (x)-\frac{2}{3 y}-\frac{2}{9 y^{2}}-\frac{44}{405 y^{3}}-\frac{26}{405 y^{4}}+\frac{40}{27 y^{5}}\right. \\
& \left.+\frac{179968}{18225 y^{6}}+\frac{4727552}{127575 y^{7}}+\frac{3436796}{32805 y^{8}}+\frac{5492621728}{22143375 y^{9}}+\ldots\right] \\
& +\ln (2)-2 \ln (y)-\ln (x) .
\end{aligned}
$$

Now we must consider $S^{(k)}(\tilde{z})$. By direct expansion, this gives the following expressions (again we provide only the first few terms). We must use up to six derivatives to get a sufficient precision (of order $x^{-2}$ ) in the Saddle integrals.

$$
\begin{aligned}
S^{(2)}(\tilde{z}) & =\frac{1}{x}\left[\frac{4}{y^{4}}+\frac{16}{3 y^{5}}+\ldots\right] \\
& +\frac{1}{x^{2}}\left[-\frac{12}{y^{4}}-\frac{40}{3 y^{5}}+\ldots\right] \\
& +\frac{1}{x^{3}}\left[\frac{12}{y^{4}}+\frac{8}{y^{5}}+\ldots\right] \\
& +\frac{1}{x^{4}}\left[\frac{-4}{y^{4}}+\ldots\right] \\
& +\mathcal{O}\left(\frac{1}{x^{5} y^{4}}\right)
\end{aligned}
$$

Note that, with $z=\tilde{z} e^{\mathbf{i} \theta}$, this leads to

$$
S^{(2)}(\tilde{z}) \frac{(z-\tilde{z})^{2}}{2} \sim-\frac{1}{2} n^{\alpha} \theta^{2} .
$$




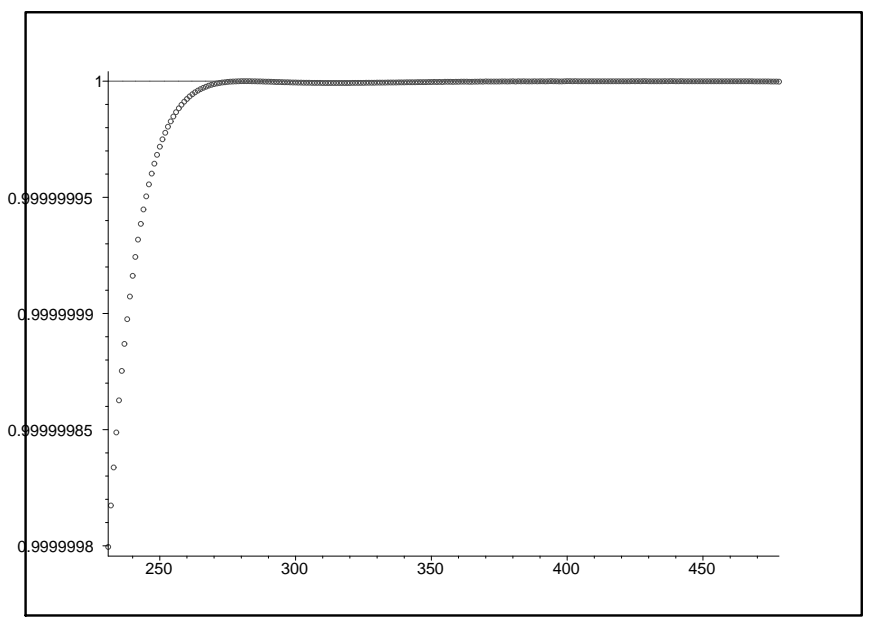

Fig. 5: $G_{n}(z n) / G_{n}(\tilde{z}), n=500$, as function of $j$

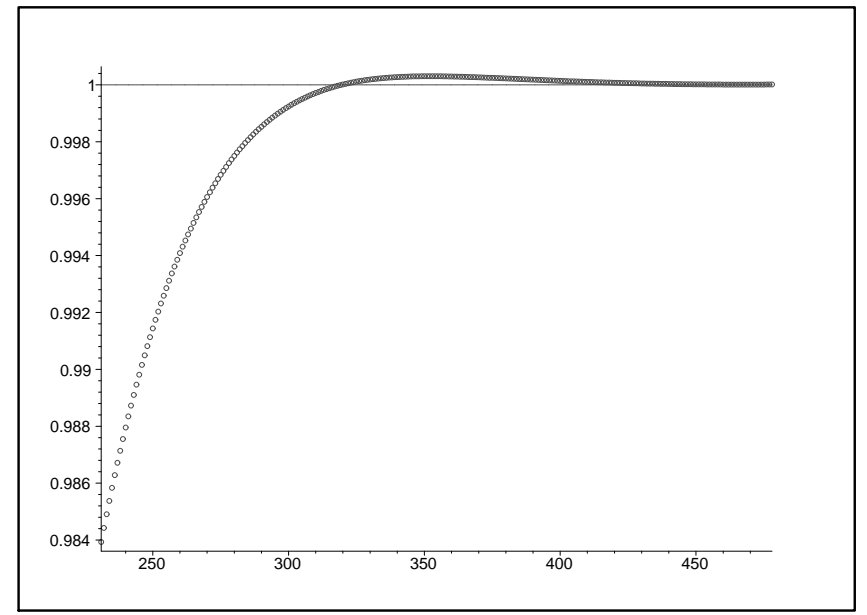

Fig. 6: The quotient of the expression $\sqrt{8}$ and $S^{(2)}(\tilde{z})$ as function of $j, n=500$ 


$$
\begin{aligned}
S^{(3)}(\tilde{z}) & =\frac{1}{x^{2}}\left[-\frac{32}{y^{6}}+\ldots\right] \\
& +\frac{1}{x^{3}}\left[\frac{128}{y^{6}}+\ldots\right] \\
& +\frac{1}{x^{4}}\left[-\frac{192}{y^{6}}+\ldots\right] \\
& +\frac{1}{x^{5}}\left[\frac{128}{y^{6}}+\ldots\right] \\
& +\mathcal{O}\left(\frac{1}{x^{6} y^{6}}\right), \\
S^{(4)}(\tilde{z}) & =\frac{1}{x^{3}}\left[\frac{288}{y^{8}}+\ldots\right] \\
& +\frac{1}{x^{4}}\left[-\frac{1440}{y^{8}}+\ldots\right] \\
& +\frac{1}{x^{5}}\left[\frac{2880}{y^{8}}+\ldots\right] \\
& +\frac{1}{x^{6}}\left[-\frac{2880}{y^{8}}+\ldots\right] \\
& +\mathcal{O}\left(\frac{1}{x^{7} y^{8}}\right), \\
& +\frac{1}{x^{6}}\left[\frac{268800}{y^{12}}+\ldots\right] \\
S^{(5)}(\tilde{z}) & =\frac{1}{x^{4}}\left[-\frac{3072}{y^{10}}+\ldots\right] \\
& +\frac{1}{x^{5}}\left[\frac{18432}{y^{10}}+\ldots\right] \\
& +\mathcal{O}\left(\frac{1}{x^{6} y^{10}}\right), \\
S^{(6)}(\tilde{z}) & =\frac{1}{x^{5}}\left[\frac{38400}{y^{12}}+\ldots\right] \\
& \\
&
\end{aligned}
$$

We proceed now as in Section 2 Again, the justification of the integration procedure is given in the Appendix. This leads to

$$
\tau=\frac{y^{2} \sqrt{x}}{2}\left[u a_{1}+\frac{u^{2} a_{2}}{x^{1 / 2}}+\frac{u^{3} a_{3}}{x}+\frac{u^{4} a_{4}}{x^{3 / 2}}+\frac{u^{5} a_{5}}{x^{2}}+\mathcal{O}\left(\frac{u^{6}}{x^{5 / 2}}\right)\right] .
$$




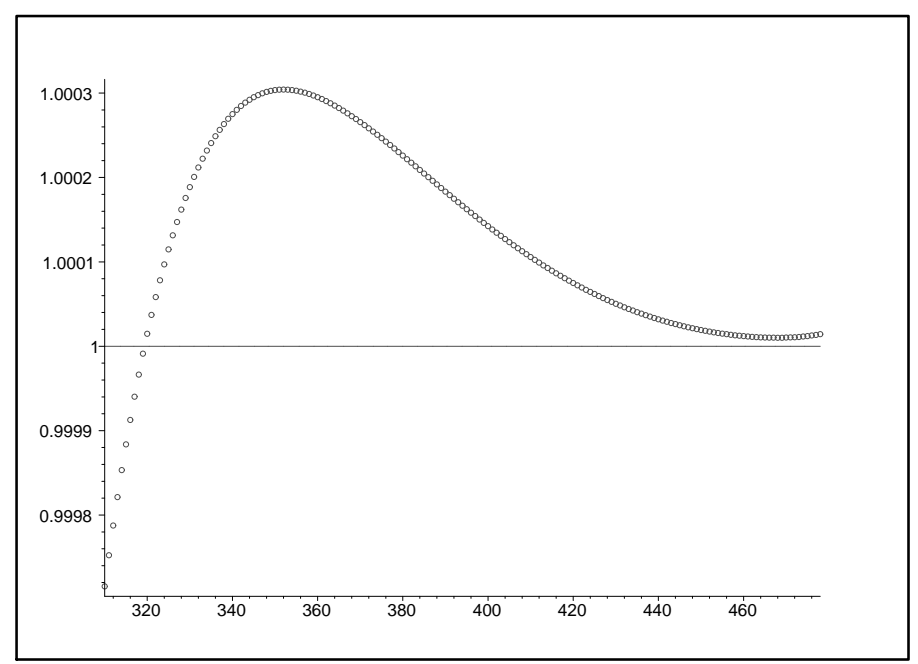

Fig. 7: The quotient of the expression 8 and $S^{(2)}(\tilde{z})$, as function of $j, n=500$. Restricted range, $\alpha \leq .84$

We give only $a_{1}$ :

$$
a_{1}=1-\frac{2}{3 y}-\frac{2}{9 y^{2}}+\ldots+\frac{1}{x}\left(\frac{3}{2}-\frac{4}{3 y}+\ldots\right)+\frac{1}{x^{2}}\left(\frac{15}{8}-\frac{7}{4 y}+\ldots\right)+\mathcal{O}\left(\frac{1}{x^{3}}\right) .
$$

This leads to

$$
\frac{1}{\sqrt{2 \pi}} \int_{-\infty}^{\infty} e^{-u^{2} / 2} \tau^{\prime}(u) d u=\frac{y^{2} \sqrt{x}}{2} T_{4}
$$

with

$T_{4}=1-\frac{2}{3 y}-\frac{2}{9 y^{2}}+\ldots+\frac{1}{x}\left(\frac{5}{12}-\frac{11}{18 y}+\ldots\right)+\frac{1}{x^{2}}\left(\frac{73}{288}-\frac{133}{432 y}+\ldots\right)+\frac{1}{x^{3}}\left(\frac{721}{576}+\ldots\right)+\mathcal{O}\left(\frac{1}{x^{4}}\right)$.

Set

This leads to

$$
T_{5}:=\frac{1}{\sqrt{2 \pi}} \frac{y^{2} \sqrt{x}}{2} e^{T_{1}}
$$

$$
\left[z^{j}\right] \phi_{n}(z) \sim T_{5} T_{3} T_{4}
$$

We can of course combine $T_{3}$ and $T_{4}$ :

$$
\begin{aligned}
T_{6} & :=T_{3} T_{4}=1-\frac{3}{3 y}-\frac{1}{18 y^{2}}-\frac{1}{30 y^{3}}+\frac{17207}{3240 y^{4}}+\ldots+\frac{1}{x}\left(-\frac{1}{12}+\frac{1}{36 y}-\frac{35}{216 y^{2}}+\frac{15029}{3240 y^{3}}+\ldots\right) \\
& +\frac{1}{x^{2}}\left(\frac{1}{288}-\frac{1}{864 y}+\frac{3527}{5184 y^{2}}+\ldots\right)+\mathcal{O}\left(\frac{1}{x^{3}}\right) .
\end{aligned}
$$




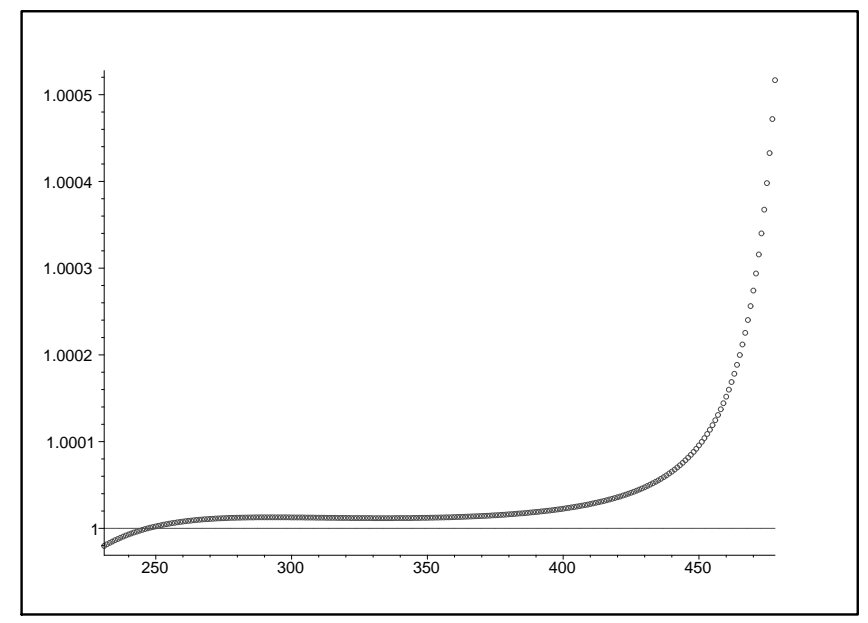

Fig. 8: The quotient $\left[z^{j}\right] \phi_{n}(z) / T_{8}$, two terms in $T_{4}$, as function of $j, n=500$

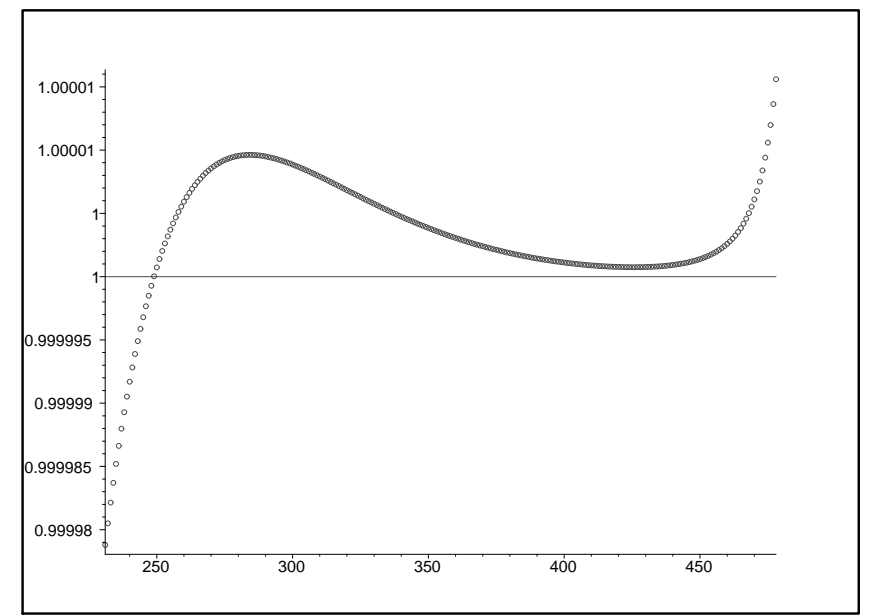

Fig. 9: The quotient $\left[z^{j}\right] \phi_{n}(z) / T_{8}$, three terms in $T_{4}$, as function of $j, n=500$ 
Let us consider the precision of our asymptotics.

The quality of asymptotic $(6)$ is given in Figure 3 and 4 for $n=500$, and $x \in\left[\sqrt{n}, n^{0.9}\right]$ (first range) so that $y \in\left[n^{0.1}, \sqrt{n}\right]$. For some values of $j=n-x$, we show $\tilde{z} / z n$, where, as mentioned, $z n$ is the numerical solution of $S^{\prime}(z n)=0$. In the full range $j \in\left[n-n^{0.9}, n-\sqrt{n}\right]$, the precision is of order $10^{-5}$, in a restricted range, the precision is of order $10^{-6}$.

Also a comparison of $G_{n}(\tilde{z})$ and $G_{n}(z n)$ is given in Figure 5 , showing again a precision of order $10^{-6}$.

To check the quality of asymptotic (6), we give in Figure 6 the comparison between the expression (8) and $S^{(2)}(\tilde{z})$. The precision is of of order $10^{-2}$.

In a restricted range, given in Figure 7 , the precision is of order $10^{-4} . \alpha \leq 0.84$ in this range.

We have made several experiments with (10), with $n$ up to 500 . The result is unsatisfactory, only values of $x$ of order $\sqrt{n}$ give reasonable results. Also using $e^{T_{2}}$ instead of $T_{3}$ does not improve the precision. Actually, only very large values of $n$ lead to good precision. So we turn to another formulation: instead of using $e^{T_{1}} T_{3}$ for $e^{S(\tilde{z})}$, we plug directly $\tilde{z}$ into $G_{n}(z)$, ie we set

$$
T_{7}=G_{n}(\tilde{z})
$$

leading to

$$
\left[z^{j}\right] \phi_{n}(z) \sim \frac{1}{\sqrt{2 \pi}} \frac{y^{2} \sqrt{x}}{2} T_{7} T_{4}=: T_{8} \text { say. }
$$

For $n=500$, using two and three terms in $T 4$, we give in Figures 8 and 9 the quotient $\left[z^{j}\right] \phi_{n}(z) / T_{8}$. The precision is of order $10^{-5}$.

\section{Conclusion}

Using an almost mechanized program in Maple, we have obtained some asymptotic expressions for Stirling numbers in central and non-central regions. We intend to use these techniques in other non-central ranges.

\section{Acknowledgement}

The pertinent comments of the referee led to improvements in the presentation.

\section{A Appendix. Justification of the integration procedure}

\section{A.1 The central region}

We proceed as in Flajolet and Sedgewick [3], ch.VIII. We can choose here $\tilde{z}=1$. This leads, with $z=e^{\mathbf{i} \theta}$, to

$$
S(z) \sim S_{0}(z)+\mathcal{O}(\sqrt{\ln (n)} \theta)+\text { constant term },
$$


with

$$
\begin{aligned}
S_{0}(z) & =\sum_{k=0}^{n-1} \ln \left[e^{\mathbf{i} \theta}+k\right]-H_{n} \mathbf{i} \theta \\
& \sim \sum_{k=0}^{n-1} \frac{1}{1+k}\left[e^{\mathbf{i} \theta}-1\right]-\frac{1}{2} \sum_{k=0}^{n-1}\left[\frac{1}{1+k}\left[e^{\mathbf{i} \theta}-1\right]^{2}-H_{n} \mathbf{i} \theta+\mathcal{O}\left(\theta^{3}\right)\right. \\
& \sim H_{n}\left[e^{\mathbf{i} \theta}-1-\mathbf{i} \theta\right]+\mathcal{O}\left(\theta^{2}\right) .
\end{aligned}
$$

Set

$$
h(\theta):=e^{\mathbf{i} \theta}-1-\mathbf{i} \theta .
$$

We have

which conforms to 3 .

$$
h(\theta) \sim-\frac{\theta^{2}}{2}
$$

The function $h(\theta)$ is the same as in [3], Ex.VIII.3, which proves the validity of our integration procedure: we use here $H_{n} \sim \ln (n)$ instead of $n$. The complete asymptotic expansion is justified as in [3], Ex.VIII.4.

\section{A.2 The non-central region}

We choose here $\tilde{z}=\frac{n y}{2}=\frac{n^{2-\alpha}}{2}:=\delta$, say. We have

$$
\begin{aligned}
& \frac{1}{2}<\alpha<1, \\
& n^{\alpha}=\frac{n^{2}}{2 \delta}, \\
& n^{2} \gg \delta \gg n \gg n^{\alpha} .
\end{aligned}
$$

Set $z=\delta e^{\mathbf{i} \theta}$, this leads, with Euler-Maclaurin formula, with the first correction (the other corrections are negligible), to

$$
\begin{aligned}
S(z) & \sim \sum_{k=0}^{n-1} \ln \left[\delta e^{\mathbf{i} \theta}+k\right]-\left(n-n^{\alpha}\right) \mathbf{i} \theta-\left(n-n^{\alpha}\right) \ln (\delta) \\
& \sim \ln \left[n+\delta e^{\mathbf{i} \theta}\right]\left[n+\delta e^{\mathbf{i} \theta}\right]-n-\delta e^{\mathbf{i} \theta} \ln \left[\delta e^{\mathbf{i} \theta}\right]-\left[n-\frac{n^{2}}{2 \delta}\right](\mathbf{i} \theta+\ln (\delta))-\frac{1}{2} \ln \left[n+\delta e^{\mathbf{i} \theta}\right]+\frac{1}{2} \ln \left[\delta e^{\mathbf{i} \theta}\right] .
\end{aligned}
$$

Set now $n=\rho \delta, \rho=2 n^{\alpha-1} \ll 1$ and expand wrt $\rho$. This gives

$$
\begin{aligned}
S(z) & \sim \rho\left[-\frac{1}{2} e^{-\mathbf{i} \theta}\right] \\
& +\rho^{2}\left[\delta \frac{1+\mathbf{i} \theta e^{\mathbf{i} \theta}}{2 e^{\mathbf{i} \theta}}+\frac{1}{4} e^{-2 \mathbf{i} \theta}+\frac{1}{2} \delta \ln (\delta)\right] \\
& +\rho^{3}\left[-\frac{\delta}{6} e^{-2 \mathbf{i} \theta}-\frac{1}{6} e^{-3 \mathbf{i} \theta}\right] \\
& +\mathcal{O}\left(\delta \rho^{4}\right) .
\end{aligned}
$$


Note that the dominant constant contribution is given by $\frac{1}{2} \rho^{2} \delta \ln (\delta)=(2-\alpha) n^{\alpha} \ln (n)$, which conforms to 7 . The first term gives a variable part $\mathcal{O}(\rho)$. The second term gives a variable part $2 n^{\alpha} h(\theta)+\mathcal{O}\left(\rho^{2}\right)$, with

$$
h(\theta):=\frac{1+\mathbf{i} \theta e^{\mathbf{i} \theta}}{2 e^{\mathbf{i} \theta}} .
$$

The third term give $\mathcal{O}\left(n^{2 \alpha-1}\right) \ll n^{\alpha}$. Note that $2 n^{\alpha} h(\theta) \sim-\frac{1}{2} n^{\alpha} \theta^{2}$, which conforms to 9 . The function $\left|e^{h(\theta)}\right|=e^{\cos (\theta) / 2}$ is unimodal with peak at 0 and $h(0)=1 / 2$. Let us introduce a splitting value $\theta_{0}$ such that $n^{\alpha} \theta_{0}^{2} \rightarrow \infty, n^{\alpha} \theta_{0}^{3} \rightarrow 0, n \rightarrow \infty$. For instance, we choose $\theta_{0}=n^{\beta}, \beta=-\frac{5 \alpha}{12}$. By unimodality property of the cosine, the tail integral

is such that

$$
K_{n}^{(1)}:=\int_{\theta_{0}}^{2 \pi-\theta_{0}} e^{2 n^{\alpha}(h(\theta)-1 / 2)} d \theta
$$

$$
\left|K_{n}^{(1)}\right|=\mathcal{O}\left(e^{n^{\alpha}\left[\cos \left(\theta_{0}\right)-1\right]}\right)=\mathcal{O}\left(e^{-C n^{\alpha / 6}}\right)
$$

for some $C>0$. The tail integral is exponentially small.

As $h(\theta) \sim-\frac{\theta^{2}}{4}$, the central approximation and the tail completion are immediate.

\section{References}

[1] E.A. Bender. Central and local limit theorems applied to asymptotics enumeration. Journal of Combinatorial Theory, Series A, 15:91-111, 1973.

[2] R. Chelluri, L. B. Richmond, and N. M. Temme. Asymptotic estimates for generalized Stirling numbers. Technical report, CWI, MAS-R9923, 1999.

[3] P. Flajolet and R. Sedgewick. Analytic combinatorics. Cambridge University press, 2009.

[4] R. L. Graham, M. Grötschel, and L. Lovász. Handbook of Combinatorics, vol. 2. Elsevier, 1995.

[5] D. B. Grünberg. On asymptotics, Stirling numbers, Gamma function and polylogs. Results in Mathematics, 49(1):89-125, 2006.

[6] H. K. Hwang. Asymptotic expansions for the Stirling numbers of the first kind. Journal of Combinatorial Theory, Series A, 71(2):343-351, 1995.

[7] H.K. Hwang. Théorèmes limites pour les structures aléatoires et les fonctions arithmétiques. 1994. Thèse, Ecole Polytechnique de Palaiseau.

[8] H.K. Hwang. On convergence rates in the central limit theorems for combinatorial structures. European Journal of Combinatorics, 19:329-343, 1998.

[9] A. Kyriakoussis and M. G. Vamvakari. On asymptotic for the signless noncental q-Stirling numbers of the first kind. Studies in Applied Mathematics, 117:191-213, 2006.

[10] L. Moser and M. Wyman. Asymptotic development of the Stirling numbers of the first kind. Journal of the London Mathematical Society, 33:133-146, 1958.

[11] N.M. Temme. Asymptotic estimates of Stirling numbers. Studies in Applied Mathematics, 89:233-243, 1993.

[12] A. N. Timashev. On asymptotic expansions of Stirling numbers of the first and second kind. Discrete Mathematics and Applications, 8(5):533-544, 1998.

[13] E. G. Tsylova. Probabilistic methods for obtaining asymptotic formulas for generalized Stirling numbers. Discrete Mathematics and Applications, 75(2):1607-1614, 1995.

[14] H. S. Wilf. The asymptotic behavior of the Stirling numbers of the first kind. Journal of Combinatorial Theory, Series A, 64:344-349, 1993. 